\title{
Species richness and composition assessment of spiders in a Mediterranean scrubland
}

\author{
Pedro Cardoso - Sérgio S. Henriques - Clara Gaspar - Luis C. Crespo • \\ Rui Carvalho $\cdot$ Jesper B. Schmidt $\cdot$ Pedro Sousa $\cdot$ Tamás Szüts
}

Received: 9 May 2007 / Accepted: 21 September 2007

(C) Springer Science+Business Media B.V. 2007

\begin{abstract}
Intensive fieldwork has been undertaken in Portugal in order to develop a standardized and optimized sampling protocol for Mediterranean spiders. The present study had the objectives of testing the use of semi-quantitative sampling for obtaining an exhaustive species richness assessment of spiders and testing the effects of day, time of day, collector and sampling method on the collected species richness and composition of a Mediterranean scrubland. The collecting summed 224 samples corresponding to one person-hour of effective fieldwork each. In total, 115 species were captured, of which 110 were recorded inside a delimited one-hectare plot, corresponding to more than $70 \%$ of the about 160 estimated species. Although no estimator reached the asymptote, the Michaelis-Menten curve behaviour indicates that the estimated richness should be accurate. Most different sampling approaches (day, time of day, collector and sampling method) were found to
\end{abstract}

P. Cardoso · J. B. Schmidt · T. Szüts

Natural History Museum of Denmark, Zoological Museum,

University of Copenhagen, Universitetsparken 15, Copenhagen

2100, Denmark

P. Cardoso

Centre for Macroecology, University of Copenhagen,

Universitetsparken 15, Copenhagen 2100, Denmark

P. Cardoso $(\bowtie) \cdot$ C. Gaspar

Agricultural Sciences Department - CITA-A, University of

Azores, Terra-Chã, Angra do Heroismo 9701-851, Portugal

e-mail: pcardoso@ennor.org

S. S. Henriques · R. Carvalho

Department of Biology, University of Évora, Núcleo da Mitra, Evora 7002-554, Portugal

S. S. Henriques

Institute of Botany and Zoology, Masaryk University, Kotlarska

2, Brno 61137, Czech Republic influence richness, abundance or composition of the samples to some extent, although sampling method had the strongest influence whereas "collector" showed no effect at all. The results support the idea that the only variables that need to be controlled in similar protocols are the sampling methods and the time of day when each method is executed. We conclude that populations in structurally simple habitats present narrower peaks of adult abundance, which implies higher percentages of juveniles in samples. Finally, results also indicate that habitats with a relatively simple structure like scrublands may require as much sampling effort, in order to reach similar proportions of captured species in relation to the estimated richness, as habitats that are much more complex.

Keywords Araneae - Biodiversity assessment . Estimators $\cdot$ Inventory $\cdot$ Sampling protocol

C. Gaspar

Biodiversity and Macroecology Group, Department of Animal and Plant Sciences, University of Sheffield, Sheffield S10 2TN, UK

L. C. Crespo

Department of Zoology, Faculty of Sciences and Technology, University of Coimbra, Largo da Se Nova, Coimbra 3000,

Portugal

P. Sousa

CIBIO, Centro de Investigação em Biodiversidade e Recursos Genéticos, Campus Agrário de Vairão, Vairao 4485-661, Portugal

P. Sousa

Departamento de Zoologia e Antropologia, Faculdade de Ciências da Universidade do Porto, Porto 4099-002, Portugal 


\section{Introduction}

Arthropods, as a megadiverse group, have always presented a formidable challenge for taxonomists, ecologists and conservationists alike. Their sheer numbers make it usually impossible to fully assess their richness and geographical patterns, even in restricted taxa or geographical areas. In a world where global biodiversity is diminishing, rapid biodiversity assessment (RBA) programs have been developed in order to try to gather as much information as possible with the scarce time and resources usually available for the task (e.g. Duelli 1997; Jones and Eggleton 2000). Such programs usually have two main issues, comparability and efficiency. Comparability between areas, instances or even taxa, can only be guaranteed by the use of standardized sampling protocols. Efficiency, so that scarce resources are not wasted, can only be guaranteed by the optimization of sampling protocols.

As a megadiverse taxon, spiders share these constraints with most other arthropods. Despite their diversity, importance in ecosystems, and the fact that probably many risk extinction or are already extinct, our enormous knowledge gap does not allow knowing the true extent of these considerations. Yet, no standardized and optimized protocols have yet been proposed for spiders in natural habitats.

Most studies involving spider diversity assessment have used a limited number of methods, leaving many species undetected due to the failure of covering many microhabitats. Spiders are very diverse in their ways of life, and sampling them requires a combination of methods. In addition, many sampling programs neglect to consider the time of day or collector experience as important variables. For spiders, complete protocols or sampling packages that gather information from a series of semi-quantitative methods have proved to be the most effective (Coddington et al. 1991). Exhaustive sampling protocols directed at spiders, mainly based on semi-quantitative methods, have been trialled in several parts of the world, i.e. Bolivia (Coddington et al. 1991), Peru (Silva and Coddington 1996), the USA (Coddington et al. 1996; Dobyns 1997; Toti et al. 2000), Tanzania (Sørensen et al. 2002) and Denmark (Scharff et al. 2003). However, they were never fully standardized and optimized.

From 2001 to 2006, systematic fieldwork has been undertaken in Portugal in order to reach a RBA protocol for Mediterranean spiders (e.g. Cardoso et al. 2004a, b, 2007). The work now presented focused on a scrubland area in southern Portugal representative of this habitat type. The objectives were to determine if it was possible to conduct an exhaustive semi-quantitative sampling of the spider community present in the habitat at the time of sampling, and which factors (day, time of day, collector and sampling method) could influence the collecting efficiency and the composition of samples.

\section{Methods}

Study site

The study was developed in a scrubland, located in Pomarão, Vale do Guadiana Nature Park, in southeastern Portugal, at an altitude of $120 \mathrm{~m}\left(\mathrm{~N} 37^{\circ} 34.500\right.$, W $007^{\circ} 32.100$ ). The site was dominated by a variety of sclerophilous plants, including rockrose (Cistus sp.), lavender (Lavandula sp.), gorse (Ulex sp.) and plants that remained from the former agricultural use of the site or from surrounding areas, mainly oat (Avena sativa L.). No plants surpassed the height of $1 \mathrm{~m}$, and in the majority of the area, the cover was much lower. The density of bushes was relatively low, with large areas of bare ground being only covered by oat and low herbaceous vegetation or even rocky patches. No trees were present in the delimited sampling area, and only a few holm oaks (Quercus ilex L.) were scattered in the region. Litter had a very thin, dry layer, when existent. Average minimum temperature of the region in January is $6^{\circ} \mathrm{C}$ and maximum temperature in August is $31^{\circ} \mathrm{C}$, although peaks above $40^{\circ} \mathrm{C}$ are common. Annual mean temperature is $17^{\circ} \mathrm{C}$ and precipitation is $500 \mathrm{~mm}$, mostly occurring during autumn and winter months and with almost null precipitation during summer.

\section{Design of the study}

We delimited a square sampling plot $100 \mathrm{~m}$ wide (1 ha), where most of the sampling effort was applied. Additional effort was made around the sampling plot, up to a distance of about $100 \mathrm{~m}$ and an overall sampling area of about $10 \mathrm{ha}$. Sampling was made from May 9 to 23 of 2006, although most effort was concentrated from May 19 to 22 (hereafter days 1 to 4). This period was chosen because it is the richest annually in Mediterranean areas (Cardoso et al. 2007).

In total, 224 samples were taken. Each sample consisted in one person-hour of work independently of the method. From these, 128 samples were made inside the delimited plot over 4 days, both day and night, by eight collectors, using two different methods. Sampling followed a balanced design, that is, the same effort was applied for each sampling day, time of day, collector and sampling method.

Sampling days

Since sampling was concentrated in 4 days, in a relatively simple habitat with a scarce variety of microhabitats, this 
continuous collecting was considered sufficient to provide a close to complete representation of the community. Besides, both the trampling of the ground and the continuous sweeping of plants certainly disturbed the habitat to a certain extent. We wanted to know if such sampling and disturbance would cause the number of species and specimens to decrease from the beginning to the end of the fieldwork and if the species composition would change with time.

Time of day

In both tropical (e.g. Sørensen et al. 2002) and Mediterranean (Cardoso et al. unpublished results) forests, night sampling was found to perform considerably better than day sampling, as most spiders are night active. We wanted to test if night collecting would differ in the richness, abundance and composition of species from the day collecting and if one of the periods would be preferred. Therefore, each collector made 2 day and two night samples inside the plot every day. Headlamps were used for night collecting.

\section{Collectors}

Contrary to other occasions (e.g. Coddington et al. 1996), it was not possible to divide the collectors according to experience, as they were very similar in this respect. However, all the eight collectors chosen for this protocol could be analysed independently.

\section{Sampling methods}

\section{Ground collection}

This method usually consists of collecting all spiders found below knee-level (Coddington et al. 1996; Sørensen et al. 2002; Scharff et al. 2003). In this habitat, as aerial searching was not one of the chosen methods and that the plants surpassing knee-level were uncommon, we have also collected above it. This did not require an increase in effort because vegetation was sparse. The specimens were captured with hand, forceps, pooter or brush and immediately transferred to $70 \%$ ethanol. Eighty samples were collected, 64 of which inside the plot.

\section{Sweep}

We used a round sweep net, with a diameter of $40 \mathrm{~cm}$, $60 \mathrm{~cm}$ deep and with a one-meter handle to sweep all vegetation. Eighty samples were collected, 64 of which inside the plot.

\section{Pitfall}

Two hundred and fifty six pitfall traps were used next to the delimited plot in a square of 16 by 16 traps. Each trap was 5 meters from the nearest traps. A sample was considered as a 2 by 2 square of four traps so that we had 64 samples in total. The clumping of traps made individual sample effort reasonably comparable with time-based samples (being the effort applied to put and collect four traps equivalent to one person-hour of work) and reduced stochastic heterogeneity among samples. The traps were set outside but next to the plot to avoid interference with the collectors. Pitfall traps were left in the field for 2 weeks, from May 9 to 23. Two plastic cups, $8 \mathrm{~cm}$ wide at the top, $12 \mathrm{~cm}$ high, and $33 \mathrm{cl}$ capacity were used for each trap, one inside the other for easy emptying. Each cup was filled two-thirds full with a preservative liquid, an anti-freeze solution containing $50 \%$ of ethylene glycol. We covered every trap with a square wooden plate, placed about $2 \mathrm{~cm}$ above the ground.

\section{Statistical analysis}

In many of the performed analyses, we have only considered the plot-based samples, which were the samples that fully complied with a balanced design. Pitfall traps, with an equivalent effort, were also considered as plot-based for some of the analyses, as this method has been previously found to be very important to complement the other two methods (Cardoso et al. unpublished results).

To know if effort was enough to have a thorough representation of the community we used EstimateS software (Colwell 2005) to calculate randomized accumulation curves of observed species richness, singletons, doubletons, and several different estimators (Chao 1, Chao 2, First and second order Jackknife and Michaelis-Menten). One thousand randomizations were always used.

Inventory completeness, defined as observed species richness in relation with estimated richness, was calculated using the Chao1 estimate, so that completeness values were comparable with previous studies (Sørensen et al. 2002; Scharff et al. 2003). Sampling intensity, defined as the ratio of specimens to species, was calculated as an approximate measure of effort (Coddington et al. 1996).

To test if there were differences on abundance or species richness per sample (dependent variables) between days, times of day, collectors and sampling methods (factors), a four-way ANOVA was made for each dependent variable, 
considering no interactions. Then, a three-way ANOVA was made considering interactions, but excluding collectors as a factor and with times of day grouped in day and night periods. Abundance data were log transformed, successfully controlling the heterogeneity of variance (Zar 1984). In all cases, the posthoc Tukey HSD test was used to find which possible pairs were significantly different for each studied factor.

We used an analysis of similarity (ANOSIM by Clarke 1993; implemented at Seaby and Henderson 2004) and the Spearman rank correlation index to compare the taxonomic composition of samples of the diverse days, times of day, collectors and methods. Abundance data of species per sample were log transformed for the ANOSIM analyses, so that the most common species did not disproportionately influence the results.

For most of the calculations (except for the ANOSIM), we used the Statistica 6 package (Statsoft Inc. 2001). Sorting and identification of specimens was made by the first author. Whenever possible, identifications were made to the species level; otherwise, morphospecies were defined. Only adult specimens were considered for statistical purposes.

\section{Results}

Overall, the 224 samples included 12007 specimens, of which 3059 (25\%) were adults. These specimens represent 115 species in 83 genera and 31 families (Appendix 1).

One of these species constitutes a novelty for the Iberian Peninsula: Haplodrassus minor (O.P.-Cambridge, 1879). Two more are new for Portugal, besides the above mentioned: Singa neta (O.P.-Cambridge, 1872) and Xysticus cribratus Simon, 1885. All specimens are deposited at the Zoological Museum of the University of Copenhagen (ZMUC).

The sampling intensity was 27 individuals per species, being $29 \%$ of the species singletons (Table 1). The species accumulation curve (Fig. 1) was not quite reaching the asymptote by the end of the sampling process. The Chao 1 estimated a richness value inside the range of all the nonparametric estimators (all around 160) and was used to calculate $71 \%$ of sampling completeness (Table 1). By the end of the randomized accumulation curves, none of the estimators reached an asymptote or was close to it (Fig. 1). The only exception is the Michaelis-Menten, which showed unrealistically low estimates. The singletons and doubletons curves were still diverging by the end of the process (Fig. 1).

Inside the plot and including the pitfall traps, 10565 spiders were collected, with 2675 adults representing 110 species (Table 1). Plot-based sampling intensity was lower than the overall data, but the percentage of singletons
Table 1 Summary data for the overall captures of this study

\begin{tabular}{llll}
\hline & \multicolumn{2}{l}{ Inside sampling plot } & Total \\
\cline { 2 - 3 } & $\begin{array}{l}\text { Pitfall } \\
\text { excluded }\end{array}$ & $\begin{array}{l}\text { Pitfall } \\
\text { included }\end{array}$ \\
\hline Samples & 128 & 192 & 224 \\
Individuals (inc. juv.) & $1683(7606)$ & $2675(10565)$ & $3059(12007)$ \\
Individuals/sample & 13.1 & 13.9 & 13.7 \\
Species & 86 & 110 & 115 \\
Species/sample & 6.1 & 6.7 & 6.6 \\
Sampling intensity & 20 & 24 & 27 \\
Singletons & $23(27 \%)$ & $32(29 \%)$ & $33(29 \%)$ \\
Doubletons & $13(15 \%)$ & $10(9 \%)$ & $10(9 \%)$ \\
Estimates & & & \\
Chao 1 \pm SD & $104 \pm 9$ & $155 \pm 18$ & $163 \pm 18$ \\
Chao 2 \pm SD & $106 \pm 9$ & $163 \pm 20$ & $171 \pm 21$ \\
Jackknife 1 \pm SD & $110 \pm 5$ & $143 \pm 7$ & $149 \pm 7$ \\
Jackknife 2 & 121 & 167 & 174 \\
Michaelis-Menten & 91 & 112 & 116 \\
Completeness & $83 \%$ & $71 \%$ & $71 \%$ \\
\hline
\end{tabular}

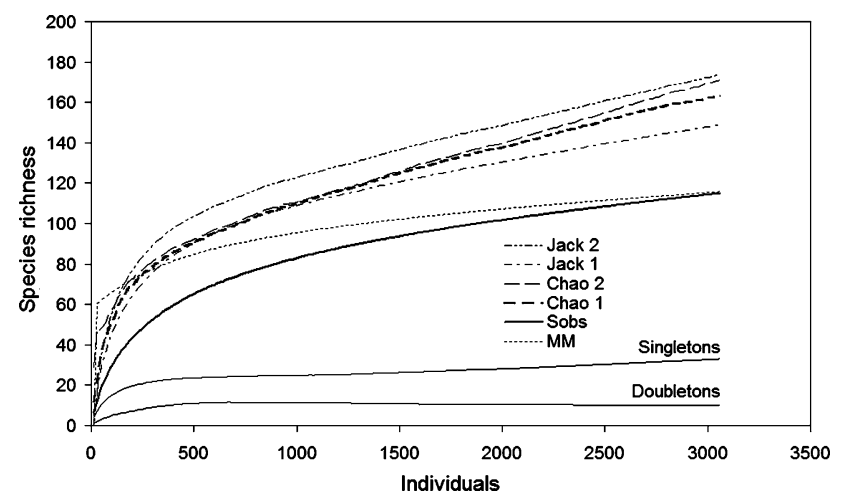

Fig. 1 Randomized accumulation curves for observed species richness, singletons, doubletons and richness estimators for all data

remained similar. Given that only a few samples were taken outside the plot, the curves and all respective values are very similar to Fig. 1.

ANOVA results indicated that only "sampling method" and "day" significantly influenced both richness and abundance per sample (Table 2). Even the "time of day", usually found to influence the productivity of sampling, did not show differences in this analysis (Table 2). On the other hand, the ANOVA results taking interactions into account revealed a significant interaction between method and period, meaning that although the overall results of day and night were similar, different methods behaved differently when comparing their day and night productivities (for individuals $F_{1,112}=6.175, P=0.014$; for species $\left.F_{1,112}=5.711, P=0.019\right)$. Analyses did not show other significant interactions. 
Table 2 Four-way ANOVAs of two different models analyzed with the plot-based sampling data

\begin{tabular}{lllll}
\hline & S.S. & d.f. & $F$ & $P$ \\
\hline Individuals & & & & \\
Day & 0.678 & 3 & 3.956 & 0.010 \\
Time of day & 0.018 & 3 & 0.103 & 0.958 \\
Collector & 0.750 & 7 & 1.876 & 0.080 \\
Method & 8.333 & 1 & 145.981 & $<0.001$ \\
Species & & & & \\
Day & 55.086 & 3 & 3.220 & 0.025 \\
Time of day & 6.648 & 3 & 0.389 & 0.761 \\
Collector & 66.305 & 7 & 1.661 & 0.126 \\
Method & 438.820 & 1 & 76.952 & $<0.001$ \\
\hline
\end{tabular}

For all models, only the main effects were calculated (no interactions). Analyses were made with either individuals $(\log 10$ transformed) or species per sample as dependent variable

Sampling days

A non-randomized collecting curve gives a better idea on the gain of species along time than a randomized curve (Fig. 2). In the present case, a steep increase in richness was observed during the first 2 days, followed by a moderate addition of species for the last two. The last day added five species to the pool, including one during the last hour (8 samples) of collecting.

The abundance and richness per sample along time showed an unusual pattern (Table 3) with the Tukey HSD test revealing differences between the second and fourth days, both in richness and abundance per sample $(P<0.043)$. The absolute numbers of abundance and richness revealed that the intermediate days, 2 and 3, presented higher values than the first and last (Table 3). However, species composition was similar for all the days, as indicated both by ANOSIM $(P>0.184$ in all paired

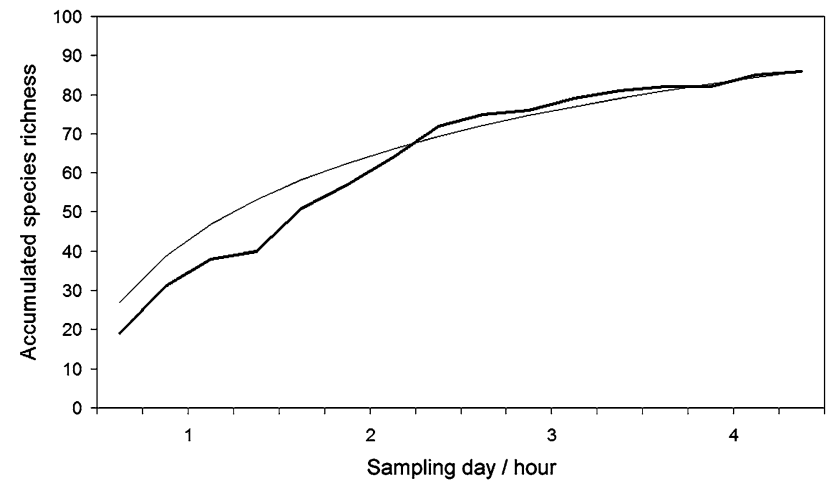

Fig. 2 Chronological accumulation curve (thick line) of species richness inside the plot, and randomized accumulation curve based on the same data (thin line). Subdivisions on the $\mathrm{x}$-axis represent one "collecting hour", each representing eight samples taken simultaneously by eight collectors
Table 3 Species richness and abundance over time

\begin{tabular}{lllll}
\hline Day & 1 & 2 & 3 & 4 \\
\hline Samples & 32 & 32 & 32 & 32 \\
Individuals & $328(19 \%)$ & $511(30 \%)$ & $528(31 \%)$ & $316(19 \%)$ \\
Individuals/sample & 10.2 & 16.0 & 16.5 & 9.9 \\
Species & $40(47 \%)$ & $67(78 \%)$ & $55(64 \%)$ & $54(63 \%)$ \\
Unique species & 2 & 13 & 8 & 5 \\
Species/sample & 5.6 & 7.0 & 6.5 & 5.4 \\
Sampling intensity & 8 & 8 & 10 & 6 \\
\hline
\end{tabular}

comparisons; full data $R=-0.007, P=0.929)$ and Spearman correlation results $\left(r_{\mathrm{s}}>0.586\right.$ and $P<0.001$ in all paired comparisons).

Time of day

Day and night samples revealed similar numbers of species and specimens per sample, either analyzing each time of day separately or grouped in day and night periods (Tables 2, 4). However, the absolute numbers reveal a tendency of higher abundance during the day but higher species richness during the night, including unique species (Table 4). The Spearman correlation index did not detect differences on species composition $\left(r_{\mathrm{s}}>0.393, P<0.001\right.$ in all paired comparisons). The ANOSIM, although not significantly, revealed that day and night samples were "less equal" compared to each other $(R>0.014$, $P<0.212)$ than when comparisons were made between similar periods $(R<-0.013, P>0.706)$. The full data results revealed this tendency to be close to significance (full data $R=0.008, P=0.096$ ).

\section{Collectors}

The non-significance of productivity differences between collectors (Table 2) did not reflect the apparently large differences of the total and unique species captured by each (Table 5). The species composition was similar for all collectors, as indicated both by ANOSIM $(P>0.063$ in all paired comparisons; full data $R=0.002, P=0.369$ ) and Spearman correlation results $\left(r_{\mathrm{s}}>0.437, P<0.001\right.$ in all paired comparisons).

\section{Sampling methods}

The ANOVA results revealed that methods were the most important factor to be taken into account (Table 2), with sweep and pitfall capturing a considerably larger number of 
Table 4 Species richness and abundance found at any time of the day (D1 and D2 are the two consecutive day samples, N1 and N2 the two consecutive night samples)

\begin{tabular}{|c|c|c|c|c|c|c|}
\hline & \multicolumn{4}{|c|}{ Time of Day } & \multicolumn{2}{|l|}{ Period } \\
\hline & D1 & D2 & N1 & N2 & Day & Night \\
\hline Samples & 32 & 32 & 32 & 32 & 64 & 64 \\
\hline Individuals & $471(28 \%)$ & $443(26 \%)$ & $396(24 \%)$ & $373(22 \%)$ & $914(54 \%)$ & $769(46 \%)$ \\
\hline Individuals/sample & 14.7 & 13.8 & 12.4 & 11.7 & 14.3 & 12.0 \\
\hline Species & $54(63 \%)$ & $44(51 \%)$ & $54(63 \%)$ & $53(62 \%)$ & $62(72 \%)$ & $73(85 \%)$ \\
\hline Unique species & 6 & 3 & 10 & 8 & 13 & 24 \\
\hline Species/sample & 6.2 & 5.8 & 6.4 & 6.0 & 6.0 & 6.2 \\
\hline Sampling intensity & 9 & 10 & 7 & 7 & 15 & 11 \\
\hline
\end{tabular}

Table 5 Species richness and abundance captured by each collector

\begin{tabular}{lllllllll}
\hline Collector & 1 & 2 & 3 & 4 & 5 & 6 & 7 & 8 \\
\hline Samples & 16 & 16 & 16 & 16 & 16 & 16 & 16 \\
Individuals & $223(13 \%)$ & $178(11 \%)$ & $188(11 \%)$ & $215(13 \%)$ & $165(10 \%)$ & $199(12 \%)$ & $250(15 \%)$ & $265(16 \%)$ \\
Individuals/sample & 13.9 & 11.1 & 11.8 & 13.4 & 10.3 & 12.4 & 15.6 \\
Species & $38(44 \%)$ & $32(37 \%)$ & $37(43 \%)$ & $38(44 \%)$ & $37(43 \%)$ & $46(53 \%)$ & $40(47 \%)$ & $43(50 \%)$ \\
Unique species & 2 & 0 & 2 & 3 & 4 & 6 & 3 & 6 \\
Species/sample & 6.4 & 4.7 & 5.6 & 6.2 & 5.6 & 6.6 & 6.6 & 7.1 \\
Sampling intensity & 6 & 6 & 5 & 6 & 4 & 4 & 6 \\
\hline
\end{tabular}

specimens and species per sample than ground searching (Table 6). Despite such differences per sample, the total number of species captured by each method was comparable, with all capturing $50 \%$ or more of the observed species (Table 6).

The accumulation curves for ground (Fig. 3) revealed that all the estimators were still rising considerably. Sweep was the method that presented the highest sampling intensity (Table 6) and, correspondingly, the Chao estimators were reaching the asymptote (Fig. 3). With this method, and similarly to what happens with the overall captures, the Michaelis-Menten estimator was barely higher than the observed richness. With intermediate values of sampling intensity, pitfall Chao estimates seemed to have approached the asymptote (Fig. 3).

Sweep and pitfall both presented a high proportion of unique species, with ground sharing many species with both methods and therefore presenting a smaller number. The ANOSIM revealed that all methods captured a different part of the community $(R>0.392, P<0.001$ in all paired comparisons, full data $R=0.628, P<0.001$ ). Pitfall and sweep even presented a strong negative correlation $\left(r_{\mathrm{s}}=-0.320, P<0.001\right)$, although pitfall and ground have presented a positive correlation $\left(r_{\mathrm{s}}=0.335, P<0.001\right)$, indicating that ground was closer to pitfall than to sweep, with which there was no correlation $\left(r_{\mathrm{s}}=0.023, P>0.05\right)$.
Table 6 Species richness and abundance per method

\begin{tabular}{llll}
\hline Method & Ground & Sweep & Pitfall \\
\hline Samples & 64 & 64 & 64 \\
Individuals & $383(14 \%)$ & $1300(49 \%)$ & $992(37 \%)$ \\
Individuals/sample & 6.0 & 20.3 & 15.5 \\
Species & $55(50 \%)$ & $58(53 \%)$ & $65(59 \%)$ \\
Unique species & 9 & 24 & 24 \\
Species/sample & 4.3 & 8.0 & 7.9 \\
Sampling intensity & 7 & 22 & 15 \\
Singletons & $19(35 \%)$ & $22(38 \%)$ & $24(37 \%)$ \\
Doubletons & $5(9 \%)$ & $9(16 \%)$ & $7(11 \%)$ \\
Estimates & & & \\
Chao 1 \pm SD & $84 \pm 15$ & $81 \pm 11$ & $100 \pm 16$ \\
Chao 2 \pm SD & $76 \pm 11$ & $83 \pm 12$ & $95 \pm 14$ \\
Jackknife 1 \pm SD & $74 \pm 4$ & $81 \pm 5$ & $89 \pm 6$ \\
Jackknife 2 & 85 & 94 & 104 \\
Michaelis-Menten & 66 & 59 & 68 \\
Completeness & $65 \%$ & $72 \%$ & $65 \%$ \\
\hline
\end{tabular}

Methods and time of day interaction

Apparent dissimilarities regarding the different productivity of methods according to time of day were present (Table 7). Ground seemed to be more productive during 
Fig. 3 Randomized accumulation curves for observed species richness, singletons, doubletons and richness estimators for all methods, inside the sampling plot
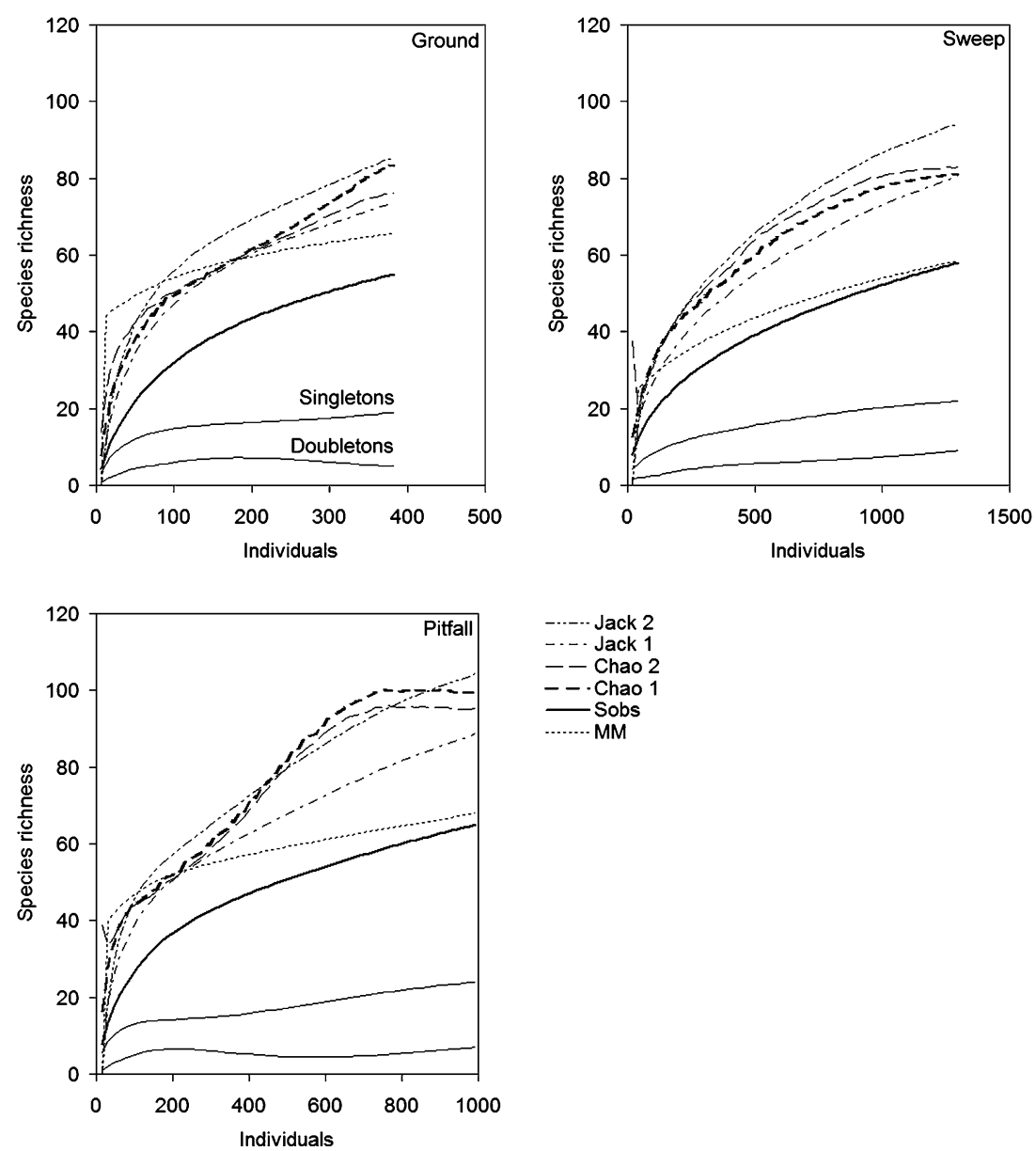

Table 7 Species richness and abundance captured by each combination of method and time of the day

\begin{tabular}{|c|c|c|c|c|}
\hline & \multicolumn{2}{|l|}{ Ground } & \multicolumn{2}{|l|}{ Sweep } \\
\hline & Day & Night & Day & Night \\
\hline Samples & 32 & 32 & 32 & 32 \\
\hline Individuals & $165(10 \%)$ & $218(13 \%)$ & $749(45 \%)$ & $551(33 \%)$ \\
\hline Individuals/sample & 5.2 & 6.8 & 23.4 & 17.2 \\
\hline Species & $35(41 \%)$ & $48(56 \%)$ & $43(50 \%)$ & $40(47 \%)$ \\
\hline Unique species & 2 & 12 & 11 & 10 \\
\hline Species/sample & 3.7 & 4.9 & 8.3 & 7.6 \\
\hline Sampling intensity & 5 & 5 & 17 & 14 \\
\hline Singletons & $12(34 \%)$ & $19(40 \%)$ & $18(42 \%)$ & $16(40 \%)$ \\
\hline Doubletons & $3(9 \%)$ & $8(17 \%)$ & $2(5 \%)$ & $8(20 \%)$ \\
\hline \multicolumn{5}{|l|}{ Estimates } \\
\hline Chao $1 \pm \mathrm{SD}$ & $52 \pm 11$ & $67 \pm 10$ & $94 \pm 26$ & $53 \pm 8$ \\
\hline Chao $2 \pm \mathrm{SD}$ & $43 \pm 6$ & $66 \pm 10$ & $126 \pm 41$ & $56 \pm 9$ \\
\hline Jackknife $1 \pm$ SD & $47 \pm 4$ & $67 \pm 4$ & $61 \pm 4$ & $56 \pm 5$ \\
\hline Jackknife 2 & 52 & 78 & 78 & 66 \\
\hline Michaelis-Menten & 48 & 66 & 46 & 43 \\
\hline Completeness & $67 \%$ & $72 \%$ & $46 \%$ & $75 \%$ \\
\hline
\end{tabular}

All percentages are relative to the plot-based sampling, excluding pitfall traps the night in absolute numbers but the Tukey HSD did not confirm the same pattern for species and specimens per sample (Table 7, $P>0.162$ ). Sweeping presented some advantages during day sampling, but this was not significant $(P>0.299)$ and similar in absolute values (Table 7).

Although day and night taxonomic composition was correlated $\left(r_{\mathrm{s}}>0.500, P<0.001\right)$ for both methods, it presented highly significant differences $(P<0.007$ in all paired comparisons; full data ANOSIM $R=0.458$, $P<0.001)$. Contrary to richness and abundance values, such day and night differences were higher for sweep $(R=0.363, \quad P<0.001)$ than for ground $(R=0.059$, $P=0.007)$.

\section{Discussion}

Given the limited number of methods employed, the spider sampling attained with this protocol was not as exhaustive as in other sites of the country (Table 8). Although the habitat presented the simplest vegetation structure of all sites sampled with this protocol to date, all the statistics present the lowest figures, with almost $30 \%$ of singletons and $71 \%$ of the estimated richness being captured. The 
Table 8 Comparison of all semi-quantitative sampling protocols that followed a similar strategy in Portugal

\begin{tabular}{|c|c|c|c|c|c|c|}
\hline & Arrábida & $\begin{array}{l}\text { Arrábida } \\
\text { (inside plot) }\end{array}$ & Gerês & $\begin{array}{l}\text { Gerês } \\
\text { (inside plot) }\end{array}$ & Guadiana & $\begin{array}{l}\text { Guadiana } \\
\text { (inside plot) }\end{array}$ \\
\hline Samples & 475 & 320 & 480 & 320 & 224 & 192 \\
\hline Abundance & 7423 & 5548 & 10808 & 7516 & 3059 & 2675 \\
\hline Richness & 168 & 150 & 204 & 185 & 115 & 110 \\
\hline Sampling intensity & 44 & 37 & 53 & 41 & 27 & 24 \\
\hline Singletons & $18 \%$ & $17 \%$ & $19 \%$ & $21 \%$ & $29 \%$ & $29 \%$ \\
\hline Estimated richness (Chao 1) & 188 & 162 & 232 & 213 & 163 & 155 \\
\hline Completeness & $89 \%$ & $92 \%$ & $88 \%$ & $87 \%$ & $71 \%$ & $71 \%$ \\
\hline
\end{tabular}

The Arrábida sampling was made in Quercus suber woodland of Central Portugal, the Gerês study was conducted in a mixed Quercus robur and Quercus pyrenaica habitat in Northern Portugal and the Guadiana study was made in a scrubland in Southern Portugal

sampling intensity, lower than 30 specimens per species, can provide an explanation for the relatively poor results. Even the behaviour of the estimators is not the best, with none of them reaching an asymptote when all data is considered. Nevertheless, the fact that the MichaelisMenten is almost crossing the observed curve (Fig. 1) may be relevant if this estimator properties are congruent with that observed at different occasions, which revealed that when the crossing between the two curves occurs, the Chao estimates can be considered accurate (Magurran 2004; Cardoso et al. unpublished results).

The fluctuations in richness and abundance during the course of the sampling may be due to the weather. During the second night of sampling, there was some light rain, uncommon in the region during this season. The probably more favourable conditions for many species may have caused the rise in numbers, although other unperceived factors can also be involved.

Confirming all the previously published studies (Coddington et al. 1991, 1996; Dobyns 1997; Sørensen et al. 2002; Scharff et al. 2003), the methods employed are the most important factor to be taken into account when replicating sampling in different areas. Another pattern that is corroborated by many studies is that all the most commonly employed methods are able to capture a close overall number of species, independently of their productivity per sample. The more productive methods in terms of number of specimens, in this case sweep and pitfall, however do show more accurate estimates of richness, with the curves presenting a behaviour that allows higher confidence in the conclusions. As their behaviour in sweep and pitfall was far better than the one of the other estimators, the Chao estimators should be the best for short-term semiquantitative sampling programs in delimited, relatively uniform areas.

Although an analysis by time of day or period does not differentiate differences in productivity or taxonomic composition, the analysis of method and period combined does show significant differences, especially concerning the latter. Even if the productivity is similar, sampling with the same method by day or night is so different that each combination may be regarded as a different method in itself.

Differences in productivity and sample composition between collectors could not be found. This result is encouraging for the future standardization and optimization of the protocol. It is usually possible to replicate the time of the year, the duration of sampling, the number of samples per method and time of day and a number of other factors, but it is usually not possible to guarantee the same collectors at each collecting event. This is especially critical for long-term studies that last for a number of years, when maintaining the same human resources is virtually impossible. Nevertheless, it seems desirable that some of the collectors of future sampling events have been previously enrolled in similar fieldwork, in order to guarantee a comparison of procedures.

A low percentage of adults was found in this study, $25 \%$, against the higher percentages reached in other regions of the country, always above $30 \%$ (Table 8 ). Cardoso et al. (2007) provide the explanation for such differences. The high canopy density of some habitats provides a shelter against extreme temperatures, wind, rain, or other meteorological factors for the lower layers. As a consequence, the microclimatic conditions are relatively constant throughout the year. This possibly allows the coexistence of many species as adults during longer periods, with longer optimum conditions for breeding. Without such protection, like in the open habitat studied, the optimum window for species' reproduction is much shorter and a lower percentage of overall annual adult richness is found during any season throughout the year (Cardoso et al. 2007). This logic may be extended to the abundance found during the study and also explains the low percentage of adults. These results have implications in the building of a future standardized and optimized protocol. "Simple" habitats, with no trees and therefore where only a few methods can be applied, require a higher effort per method 
in order to capture the same proportion of overall richness present at the site. Moreover, when it is possible to execute a greater variety of methods, even stochastic factors can help increase the completeness, as many species may be captured by chance alone in methods less adequate to them, and the more the methods employed the bigger the chance for this to happen. A future protocol that will be usable in any Mediterranean habitat will probably have to take this compensation of effort per method into account.

In conclusion, this study emphasizes three main points:

1) Method and time of day are the most important factors to take into account in sampling protocols;

2) Populations in structurally simple habitats present narrower peaks of adult abundance, which implies higher percentages of juveniles in samples;

3) Structurally simple habitats may require as much sampling effort as more complex habitats in order to reach the same completeness levels.

Acknowledgements $\mathrm{PC}$ is supported by the Portuguese Foundation for Science and Technology (SFRH/BPD/17351/2004/9ME3). Fieldwork in Vale do Guadiana Nature Park was supported by the Danish Bøje Benzons Foundation.

Appendix 1 List of the captured species grouped by family and respective adult abundances

Agelenidae (2 spp.)

Agelena labyrinthica (Clerck, 1757)

Tegenaria feminea Simon, 1870

Araneidae (7 spp.)

Aculepeira armida (Audouin, 1826)

Agalenatea redii (Scopoli, 1763)

Cyclosa insulana (Costa, 1834)

Hypsosinga albovittata (Westring, 1851)

Mangora acalypha (Walckenaer, 1802)

Neoscona adianta (Walckenaer, 1802)

Singa neta (O. P.-Cambridge, 1872)

Clubionidae (2 spp.)

Clubiona genevensis L. Koch, 1866

Clubiona sp.

Dictynidae (3 spp.)

Archaeodictyna consecuta (O. P.-Cambridge, 1872)

Dictyna civica (Lucas, 1850)

Lathys simplex (Simon, 1884)

Dysderidae (2 spp.)

Dysdera alentejana Ferrández, 1996

Harpactea minoccii Ferrández, 1982

Filistatidae (1 sp.)

Pritha cf. pallida (Kulczynski, 1897)

Gnaphosidae (21 spp.)

Drassodes lapidosus (Walckenaer, 1802)

Appendix 1 continued

Drassodes sp. 4

Haplodrassus macellinus (Thorell, 1871) 16

Haplodrassus minor (O. P.-Cambridge, 1879) 8

Leptodrassus albidus Simon, 1914

Leptodrassus simoni Dalmas, $1919 \quad 18$

Micaria coarctata (Lucas, 1846)

Nomisia excerpta (O. P.-Cambridge, 1872) 20

Nomisia exornata (C. L. Koch, 1839) 24

Pterotricha simoni Dalmas, 1921

Trachyzelotes bardiae (Caporiacco, 1928) 21

Trachyzelotes fuscipes (L. Koch, 1866) 23

Trachyzelotes holosericeus (Simon, 1878) 7

Zelominor algarvensis Snazell \& Murphy, 1997

Zelotes cf. gallicus Simon, 1914

Zelotes denisi Marinaro, $1967 \quad 1$

Zelotes sp. 1

Zelotes sp. 2

Zelotes sp. $3 \quad 1$

Zelotes sp. $4 \quad 2$

Zelotes sp. 5

Hahniidae (1 sp.) 1

Hahnia nava (Blackwall, 1841) 1

Linyphiidae (10 spp.) 1221

Didectoprocnemis cirtensis (Simon, 1884) 1

Diplocephalus graecus (O. P.-Cambridge, 1872) 463

Erigone dentipalpis (Wider, 1834) 1

Meioneta pseudorurestris (Wunderlich, 1980) 77

Micrargus sp. $\quad 15$

Pelecopsis bucephala (O. P.-Cambridge, 1875) 7

Pelecopsis inedita (O. P.-Cambridge, 1875) 622

Prinerigone vagans (Audouin, 1826) 27

Styloctetor romanus (O. P.-Cambridge, 1872) 7

Tenuiphantes tenuis (Blackwall, 1852) 1

Liocranidae (1 sp.) 1

Mesiotelus mauritanicus Simon, $1909 \quad 1$

Lycosidae (3 spp.) 8

Alopecosa sp. 6

Lycosa tarantula (Linnaeus, 1758) 1

Pardosa proxima (C. L. Koch, 1847) 1

Mimetidae (1 sp.) 1

Ero aphana (Walckenaer, 1802) 1

Miturgidae (2 spp.) 13

$\begin{array}{ll}\text { Cheiracanthium sp. } & 10\end{array}$

Cheiracanthium striolatum Simon, 1878

Oecobiidae (1 sp.) 125

Oecobius machadoi Wunderlich, $1995 \quad 125$

Oonopidae (2 spp.) 36

Oonops sp. $\quad 22$

Silhouettella loricatula (Roewer, 1942) 14

Oxyopidae (2 spp.) 79 
Appendix 1 continued

Oxyopes heterophthalmus (Latreille, 1804)

Oxyopes lineatus Latreille, 1806

Palpimanidae (1 sp.)

Palpimanus gibbulus Dufour, 1820

Philodromidae (3 spp.)

Philodromus cespitum (Walckenaer, 1802)

Philodromus rufus Walckenaer, 1826

Thanatus vulgaris Simon, 1870

Pholcidae (1 sp.)

Holocnemus hispanicus Wiehle, 1933

Pisauridae (1 sp.)

Pisaura mirabilis (Clerck, 1757)

Prodidomidae (1 sp.)

Prodidomus amaranthinus (Lucas, 1846)

Salticidae (14 spp.)

Aelurillus luctuosus (Lucas, 1846)

Chalcoscirtus infimus (Simon, 1868)

Cyrba algerina (Lucas, 1846)

Euophrys frontalis (Walckenaer, 1802)

Euophrys sulphurea (L. Koch, 1867)

Evarcha jucunda (Lucas, 1846)

Heliophanus cupreus (Walckenaer, 1802)

Heliophanus lineiventris Simon, 1868

Leptorchestes mutilloides (Lucas, 1846)

Menemerus semilimbatus (Hahn, 1829)

Pellenes nigrociliatus (Simon, 1875)

Salticus propinquus Lucas, 1846

Salticus scenicus (Clerck, 1757)

Thyene imperialis (Rossi, 1846)

Scytodidae (1 sp.)

Scytodes velutina Heineken \& Lowe, 1832

Segestriidae (1 sp.)

Ariadna insidiatrix Audouin, 1826

Sicariidae (1 sp.)

Loxosceles rufescens (Dufour, 1820)

Sparassidae (1 sp.)

Eusparassus dufouri Simon, 1932

Tetragnathidae (1 sp.)

Tetragnatha sp.

Theridiidae (15 spp.)

Episinus maculipes Cavanna, 1876

Episinus truncatus Latreille, 1809

Euryopis episinoides (Walckenaer, 1847)

Kochiura aulica (C. L. Koch, 1838)

Lasaeola testaceomarginata Simon, 1881

Robertus sp.

Simitidion simile (C. L. Koch, 1836)

Steatoda triangulosa (Walckenaer, 1802)

Theridiidae 1

Theridiidae 2
Appendix 1 continued

Theridiidae 3

Theridiidae 4

Theridion sp.

Theridion impressum L. Koch, 1881

159

Theridion nigropunctatum Lucas, 1846

5

Thomisidae (9 spp.)

Monaeses paradoxus (Lucas, 1846)

Runcinia grammica (C. L. Koch, 1837)

Synema globosum (Fabricius, 1775)

Thomisus onustus Walckenaer, 1805

Tmarus staintoni (O. P.-Cambridge, 1873)

Xysticus bliteus (Simon, 1875)

Xysticus bufo (Dufour, 1820)

Xysticus cf. cribratus Simon, 1885

Xysticus ferrugineus Menge, 1876

Zodariidae (3 spp.)

Zodarion jozefienae Bosmans, 1994

Zodarion merlijni Bosmans, 1994

Zodarion styliferum (Simon, 1870)

Zoridae (1 sp.)

Zora spinimana (Sundevall, 1833)

\section{References}

Cardoso P, Silva I, Oliveira NG, Serrano ARM (2004a) Higher taxa surrogates of spider (Araneae) diversity and their efficiency in conservation. Biol Conserv 117:453-459

Cardoso P, Silva I, Oliveira NG, Serrano ARM (2004b) Indicator taxa of spider (Araneae) diversity and their efficiency in conservation. Biol Conserv 120:517-524

Cardoso P, Silva I, Oliveira NG, Serrano ARM (2007) Seasonality of spiders (Araneae) in Mediterranean ecosystems and its implications in the optimum sampling period. Ecol Entomol 32:516-526

Clarke KR (1993) Non-parametric multivariate analyses of changes in community structure. Aust J Ecol 18:117-143

Coddington JA, Griswold CE, Silva-Dávila D, Peñaranda E, Larcher SF (1991) Designing and testing sampling protocols to estimate biodiversity in tropical ecosystems. In: Dudley EC (ed) The unity of evolutionary biology: Proceedings of the Fourth International Congress of Systematic and Evolutionary Biology. Dioscorides Press, Portland, pp 44-60

Coddington JA, Young LH, Coyle FA (1996) Estimating spider species richness in a southern Appalachian cove hardwood forest. J Arachnol 24:111-128

Colwell RK (2005) EstimateS: statistical estimation of species richness and shared species from samples, version 7.5

Dobyns JR (1997) Effects of sampling intensity on the collection of spider (Araneae) species and the estimation of species richness. Environ Entomol 26:150-162

Duelli P (1997) Biodiversity evaluation in agricultural landscapes: an approach at two different scales. Agric Ecosyst Environ 62:8191

Jones DT, Eggleton P (2000) Sampling termite assemblages in tropical forests: testing a rapid biodiversity assessment protocol. J Appl Ecol 37:191-203 
Magurran AE (2004) Measuring biological diversity. Blackwell Science, Oxford

Scharff N, Coddington JA, Griswold CE, Hormiga G, Bjørn PP (2003) When to quit? Estimating spider species richness in a northern European deciduous forest. J Arachnol 31:246-273

Seaby RMH, Henderson PA (2004) Community analysis package 3.0. Pisces Conservation Ltd, Lymington

Silva D, Coddington JA (1996). Spiders of Pakitza (Madre de Dios, Perú): species richness and notes on community structure. In: Wilson DE, Sandoval A (eds) The biodiversity of Southeastern Perú. Smithsonian Institution, Washington, pp 253-311
Sørensen LL, Coddington JA, Scharff N (2002) Inventorying and estimating subcanopy spider diversity using semiquantitative sampling methods in an afromontane forest. Environ Entomol 31:319-330

StatSoft Inc. (2001) STATISTICA: data analysis software system, version 6

Toti DS, Coyle FA, Miller JA (2000) A structured inventory of Appalachian grass bald and heath bald spider assemblages and a test of species richness estimator performance. J Arachnol 28:329-345

Zar JH (1984) Biostatistical analysis. Prentice Hall Inc., New Jersey 